\title{
Children's Heart Rate and Vicariously Aroused Affect in Response to Others' Differing Emotional Experiences
}

\author{
Xenia Anastassiou-Hadjicharalambous ${ }^{1,2, *}$ and David Warden ${ }^{1}$ \\ ${ }^{I}$ Department of Psychology, University of Strathclyde, Scotland and ${ }^{2}$ Department of Psychology, University of Nicosia, \\ Cyprus
}

\begin{abstract}
This study examined vicariously induced Heart Rate (HR) patterns in response to others' sadness, fear, anger and happiness, in children ( $N=44,22$ girls and 22 boys, aged 7 to 10), when confronted with a brief emotion evocative film consisting of a series of evocative episodes each of which was of at least moderate intensity. HR was consistently higher relative to baseline in response to others' fear $(p<.001)$, sadness $(p<.011)$, anger $(p<.014)$ and the positive emotion of happiness/surprise $(p<.002)$. These findings suggest that HR can reliably be used as a marker of vicariously aroused affect in response to a range of different emotions in children, given that stimulus intensity is at least moderate.
\end{abstract}

Key Words: Vicarious affective arousal, affective empathy, heart rate, children.

\section{INTRODUCTION}

Heart Rate (HR) acceleration has been associated with anxiety and active coping during stress [1-2]. There is evidence that infants as young as eight months of age show an increase in HR just before crying which suggests that HR acceleration is indicative of negative affect or stress [3]. Similarly, school-aged children and adults have shown evidence of HR acceleration when they are in distress [4]. On the other hand, because HR deceleration has been associated with the intake of information from the environment in the psychophysiological studies [1], it has been suggested that HR deceleration in sadness inducing context may be a marker of focus of attention to the needy other [5].

Such findings suggest alternative hypotheses for the patterns of HR in an emotion evocative context: The observer's HR may either increase due to his/her vicarious affective arousal or it may decrease due to his/her attention to an event (in this case an affective stimulus) that is external to self. Hoffman [6] postulates that whether HR will accelerate or decelerate depends on the intensity of the affective stimulus. If the intensity is high, the observer's experience may be aversive leading to $\mathrm{HR}$ acceleration indicative of vicarious affective arousal. If the intensity is low, the cognitive dimensions of empathy (i.e. imagining oneself in another's position) may predominate leading to a decrease in HR. Hoffman [6] further predicts that, even at low levels of stimulus intensity, where children (especially the younger ones) are concerned, more primitive modes of empathy, unmediated by cognition, will prevail, resulting to HR acceleration.

Empirical studies to determine whether HR will accelerate or decelerate in response to an emotive situation have

*Address correspondence to this author at the Department of Psychology, University of Nicosia, 46 Makedonitissas Avenue, P.O. Box 24005, 1700 Nicosia, Cyprus; Tel: +357222351274; Fax: +3572 22 353682;

E-mail: hadjicharalambous.x@unic.ac.cy produced mixed findings due to the potential influence of a variety of factors: (a) whether the stimuli have high or low intensity; (b) whether the participants are children or adults; (c) whether the stimuli are realistic or static; (d) which emotional state is involved; and (e) which moment in the timecourse of the presentation of the stimulus is observed. One line of empirical data has provided corroborative evidence of HR acceleration in response to high intensity affective (mainly fear) contexts, in both adults [7] and children [5, 810]. In contrast, HR deceleration has been found in response to low intensity, sadness-inducing contexts that typically utilized static stimuli such as pictures or slides of a crying person without any explanation of the person's situation; these results have mostly been found with adult samples [1112], but also in some child samples [12-13].

On the other hand, higher HR relative to baseline has been found in children aged 4-5 in sadness-inducing contexts that were not of high intensity with the use of videotaped scripts [14]. Importantly it was observed that when the novel stimulus was initially introduced it produced a brief deceleration indicative of attention to novel stimulus followed by acceleration indicative of vicarious affective arousal [14]. This finding may indicate that when the intensity of stimulus is at least moderate and when more ecologically valid stimuli, rather than static pictures are used, children's HR is consistently increased in response to an emotive situation.

The present study was therefore designed to examine vicariously induced HR patterns in children aged 7 to 10 years, whilst they watched an emotionally evocative film. This film featured a child who experienced a variety of emotions (sadness, happiness, fear, anger) at various points in a single storyline. Further time-locked HR patterns were observed from the instant that the emotional state of the story character was conveyed. Given that empirical investigations have not provided support for consistent differentiation of HR across differing emotional states [15], it was predicted 
that children's HR would be consistently higher relative to baseline in response to others' differing affective states across all types of evocative episodes, each of which was of at least moderate intensity. In order to further substantiate the HR patterns, children's facial expressions of emotional experience were simultaneously video-recorded to provide corroborating evidence of facially-displayed vicariously aroused affect.

\section{METHOD}

\section{Participants}

The study sample $(N=44)$ comprised 22 girls and 22 boys, aged 7 to 10 years $(M=8.7$ years $)$. All participants were of white ethnic origin and were recruited from urban and rural schools around Glasgow, UK. About $60 \%$ of the children who were invited to participate in the study eventually did so. Parental written informed consent was obtained prior to participation in the study. Children's participation was voluntary. The participating children came from families of lower-middle class socioeconomic status (SES) with a mean score on Duncan Socioeconomic Index [16] of 40 (SD $=15.38)$ that closely approximated that of the participating schools.

\section{Apparatus}

\section{Stimulus Film}

An edited version of the emotion evocative short film 'Who do you think you are' [17] (see Table 1 for description) was used as stimulus. This film depicted a series of different valence evocative episodes within a continuous story; it was appropriate for the age of the participants, and short in duration, given the nature of physiological assessments. The film, in its original form, was of 22 minutes duration, but it was edited down to 8.5 minutes by excluding extraneous scenes, and retaining only scenes which were emotionally provocative and those providing contextual coherence. The evocative episodes were selected by 15 postgraduate psychology students and 15 children aged 7 to 10 years. The postgraduate students were also requested to judge the intensity of each episode on a three-point scale (low, moderate, high). Only those episodes both indicated and eliciting agreement with respect to intensity by at least $90 \%$ of the judges were retained. The final version of the film comprised three episodes depicting sadness, two episodes depicting fear, two episodes depicting anger and one episode depicting happiness/surprise. All retained episodes were judged to be of at least moderate intensity.

\section{Heart Rate (HR)}

Electrocardiogram (ECG) data were collected every 10 milliseconds while participants watched the emotion evocative film and recorded online into a computer for subsequent computing of HR. The acquired ECG data were initially edited using the Acknowledge 3.5.3 software.

Editing the data files consisted of scanning the data for outliers relative to adjacent data (i.e. movement artifacts) and replacing them with the average of the codable beat immediately before and after the outlier.

Subsequently, HR (in beats per minute, BPM) was computed. Data were then extracted to Excel, where mean HR for baseline and each particular evocative episode were computed. Baseline HR was obtained for each child by averaging 20 seconds of neutral content in the beginning of the film, and 10 seconds in the end of the film. Baseline HR was compared to the average HR across each type of evocative episode.

\section{Table 1. Description of the Stimulus Film}

This film depicts a series of eight linked episodes and interactions involving an 11-year-old boy called Ben. Each episode is intended to elicit a particular emotional response, as follows:

1. Anger. Ben is falsely accused by a passer by of knocking down an old lady (Mrs Morton) in the street.

2. Sadness. At home, Ben is told by his mother that he cannot visit his divorced father at the weekend.

3. Anger. In response to a misunderstood message, Ben's mother is angry with him for knocking down the old lady. A row ensues about his divorced father.

4. Fear. Ben runs away from home, and hides in the local cemetery. It gets dark, a storm begins, with thunder and lightning. Sound of broken glass.

5. Fear. Ben finds Mrs Morton's cat in the storm, takes him home. Mrs Morton makes Ben take a shower, but the flame in the old gas boiler erupts dangerously.

6. Sadness. Mrs Morton comforts Ben about his absent father, and tells him that she never knew her own father, because he was a pilot killed during the war.

7. Sadness. Ben, prompted by Mrs Morton, talks movingly about his own dad, and why his parents split up.

8. Happiness/Surprise. Mrs Morton encourages Ben to phone his dad. He does so, and learns that he has a new job, and will be around much more often.

Source: Carlton Television. Health and Social Issues [17].

\section{Facial Expressions}

Facial expressions of emotional experience were collected and later scored on the basis of AFFEX facial coding system [18]. AFFEX considers only those facial movements that indicate affect, unlike other systems designed to code all facial movements [19]. Therefore, it was considered to be more efficient and less labour intensive for addressing the concerns of the present study.

AFFEX identifies eight fundamental emotional expressions (interest, joy, surprise, sadness, anger, disgust, contempt, fear) and can be used in testing from infancy through adulthood [18].

The facial expressions were scored by a coder trained for reliability on AFFEX who was blind to the hypothesis being tested. A two-minute baseline tape for each child was viewed initially to familiarise the coder with any idiosyncratic facial characteristics.

On the basis of the coding criteria given in Table $\mathbf{2}$, the coder judged the child's predominant facial expression during each of the evocative episodes.

The data of 8 children were randomly selected and checked for reliability by a second coder also trained for 
reliability on AFFEX. The inter-coder agreement was calculated using the weighted Kappa procedure. The average Kappa was .75 , ranging from .88 for happiness to .72 for sadness.

\section{Table 2. Description of AFFEX Facial Emotion Codes}

\begin{tabular}{|c|l|}
\hline Emotion & \multicolumn{1}{|c|}{ Description } \\
\hline \hline Happiness & $\begin{array}{l}\text { Forehead smooth; cheeks raised; corners of mouth } \\
\text { drawn back and up. }\end{array}$ \\
\hline Sadness & $\begin{array}{l}\text { Inner brows drawn together, vertical furrows or bulge } \\
\text { between brows; raised inner corners of upper eyelid; } \\
\text { eyes squinted; downward mouth corners; lower lip } \\
\text { pushed upward by chin muscle (chin puckers). }\end{array}$ \\
\hline Anger & $\begin{array}{l}\text { Brows sharply down and together; vertical furrows or } \\
\text { bulge between brows; nasal root broadened or bulged; } \\
\text { eyes narrowed by lowering of brow; rectangular mouth; } \\
\text { lips may be pressed together tightly. }\end{array}$ \\
\hline Fear & $\begin{array}{l}\text { Straight brows slightly raised and drawn together; hori- } \\
\text { zontal lines or bulge on forehead; nasal root narrowed; } \\
\text { eyes narrowed or squinted; eyelids raised (white shows } \\
\text { more than usual) mouth corners tight or retracted. }\end{array}$ \\
\hline Surprise & $\begin{array}{l}\text { Brows raised, bulging or long furrows completely across } \\
\text { forehead; mouth open oval or roundish. }\end{array}$ \\
\hline Neutral & $\begin{array}{l}\text { Expression as baseline. Includes both 'no expression' } \\
\text { and 'no clearly identified emotion'. }\end{array}$ \\
\hline
\end{tabular}

Note: Descriptions are based on Izard, Dougherty and Hembree [18].

\section{Procedures}

Upon obtaining approval from the Ethics committee of the University of Strathclyde, Glasgow, UK, participants were tested individually in a single laboratory session of around 30 minutes duration in a university laboratory. An hour prior to the arrival of each child and his/her parent or guardian, the recording equipment was calibrated.

Upon arrival, each child was familiarized with the setting and the experimenter and then escorted to the observation laboratory. The procedure was explained, the function of the physiological equipment was introduced and the child's consent to proceed was obtained.

The child then sat in a comfortable chair approximately 6 feet from the television screen. After the child's skin had been lightly abraded and cleaned with Skin Pure (Nihon Kohden Corporation) for reduction of the impedance of skin surface areas, three small-prejelled electrocardiogram $(E C G)$ electrodes were attached in an inverted triangle pattern in three positions: The inner wrist of the non-dominant hand, the front of the ankle of the opposite leg and, the front of the ankle of the second leg (serving as ground).

With the use of the computer-based acquiring system MPIOOSW (Biopac Systems, Inc.), the ECG signals were amplified by the ECG100 amplifier (gain set at $40 \mathrm{mV}$ ), displayed and recorded onto one channel of the instrumentation recorder for later analysis.
Once the electrodes had been attached, the child was requested to limit movement during the procedure, s/he was assured that both the guardian and the investigator would be nearby, and was left to watch the film alone and without interruption while his/her ECG and facial expressions were recorded (by a hidden camera behind a one way mirror). Sole viewing of the film was thought necessary to decrease any social demands that might attenuate the child's facial expressions [20].

For precision in the coding of the physiological responses, the recording of the ECG was synchronised to the onset of the video stimulus. This was achieved by placing an audio tone on the second channel of the VCR. This tone via specially designed equipment would be transformed into digital input, and, being connected to one channel of the acquiring equipment, would trigger the acquisition of the physiological data.

\section{RESULTS}

For data that were not normally distributed, and/or for which the equality of variance assumption was violated, median $(M d n)$ scores and the interquartile range $(I Q R)$ are presented. Between-subject differences for two-sample data were determined using the Mann-Whitney $U$ test and withinsubject differences were determined using the Wilcoxon Signed-Ranks test. For three sample data statistically significant differences were determined using the Friedman Test. Frequency data were analysed using the chi-square $\left(\chi^{2}\right)$ statistic.

HR (averaged across each type of episode) was analyzed across baseline and during exposure to the series of evocative episodes (i.e. sadness, fear, anger and happiness/surprise) of the brief film. The baseline HR ( $M d n=$ $82.5, I Q R=13.39)$ was lower relative to HR during the evocative episodes. HR was higher in the exposure to the protagonist's fear $(M d n=86.06, I Q R=19.67)$ followed by exposure to the protagonist's happiness/surprise $(M d n=$ $85.45, I Q R=17.46)$, anger $(M d n=84.97, I Q R=16.75)$ and sadness $(M d n=84.28, I Q R=17.39)$.

A Friedman test performed on HR data across the five types of episodes (baseline, sadness, anger, fear, happiness/surprise) revealed a statistically significant effect of the type of episode $\left(\chi^{2}=14.309, d f=4, p<\right.$. 006). Following the significant effect of the type of episode, pairwise comparisons using Wilcoxon Signed-Ranks tests revealed the following patterns: HR was significantly higher relative to baseline in exposure to the protagonist's fear $(z=3.326, p<.001)$, anger $(z=2.451, p<.014)$, sadness $(\mathrm{z}=2.556, p<.011)$ and happiness/surprise $(\mathrm{z}=3.058, p<.002)$. HR was significantly higher in exposure to the protagonist's fear relative to exposure to the protagonist's anger $(z=2.392, p<.017)$.

Correlational analysis (after controlling for baseline HR) revealed no correlation between child's age and HR for any type of emotional episode. Separate Mann-Whitney $U$ tests employed to determine gender effects on HR across the evocative episodes did not reveal a significant effect of gender on HR in any of the episodes.

As indicated in Table $\mathbf{3}$, children facially displayed vicarious affective responses that were congruent (either 
matching or of similar valence) to the protagonist's emotional state.

Table 3. Facially-Displayed Vicarious Responses to the Protagonist's Differing Emotional States

\begin{tabular}{|c|c|c|}
\hline Episode & Match & Statistic \\
\hline \hline Anger $(\mathrm{n}=2)$ & $58(65.9 \%)$ & $x_{(1,88)}^{2}=8.908^{*}$ \\
\hline Sadness $(\mathrm{n}=3)$ & $79(59.9 \%)$ & $x_{(1,132)}^{2}=5.121^{*}$ \\
\hline Fear $(\mathrm{n}=2)$ & $54(61.4 \%)$ & $x_{(1,88)}^{2}=4.545^{*}$ \\
\hline $\begin{array}{c}\text { Happiness/ } \\
\text { Surprise }(\mathrm{n}=1)\end{array}$ & $29(65.9 \%)$ & $x_{(1,44)}^{2}=4.455^{*}$ \\
\hline
\end{tabular}

Note: Numbers in the table indicate the frequency with which responses were elicited. Match: displayed emotion matching (or of equal valence) to the protagonist's emotion. $* p<.05$.

A separate analysis performed on HR data for those trials that elicited a congruent facial response revealed a significant increase in HR in response to the protagonist's fear $(z=$ $2.763, p<.006)$, anger $(z=2.210, p<.027)$, sadness $(\mathrm{z}=$ $2.129, p<.033)$ and happiness/surprise $(\mathrm{z}=3.058, p<.03)$. The analysis of HR data for those trials that did not elicit a congruent facial response revealed a significant increase of $\mathrm{HR}$ in response to the protagonist's happiness/surprise $(\mathrm{z}=$ $2.213, p<.027)$ but no other differences reached statistical significance.

\section{DISCUSSION}

Preliminary findings of the present investigation appear to indicate that, in children aged 7 to 10 , HR vicariously and consistently increases in response to others' fear, sadness, anger, and happiness when the intensity of the stimulus is at least moderate. These findings suggest that, in children up until the age of 10, a moderately intense stimulus is adequate to elicit vicarious affective arousal. Importantly these HR patterns were substantiated by facial data which provided corroborating evidence of simultaneous facially-displayed vicarious affective responsiveness to the protagonist's emotional experience.

Children's responses to others' fear were in line with previous investigations [5, 7-10] which reported HR to be significantly higher relative to baseline. As predicted, present findings also provide evidence for vicarious increase of HR, not only in response to another's anger and sadness, but also to the positive emotion of happiness/surprise. The present data on sadness extend upwards in age, and generalise to the population of typically-developing children, the findings which were previously reported for problem- behaviour children, and in which moderately intense affective stimuli (i.e. videotaped scripts) were used [14]. In contrast, studies that selectively used affective contexts of low intensity (i.e. static pictures or slides) reported HR deceleration in response to another's sadness [5, 12, 13, 21].

These patterns of findings taken together seem to suggest that the intensity and the type of stimuli used play a substantial role in HR patterns. In moderate and high intensity affective contexts in which quite realistic experimental inductions are utilised, HR is vicariously increased. In such contexts HR increase is indicative of vicariously aroused affect and it might be interpreted as evidence of the affective dimensions of empathy predominating over the cognitive [6].

On the other hand, in low intensity affective contexts in which static stimuli are used, the evidence provides support to the conjecture that HR decelerates. This deceleration might be interpreted as evidence of the predominance of cognitive dimensions of empathy over the affective ones [6]. However, a second explanation for HR deceleration in low intensity static sadness context is that HR deceleration indicates attention to a novel stimulus rather than any empathic reaction. Support for this explanation comes from studies reporting an initial brief $\mathrm{HR}$ deceleration followed by $\mathrm{HR}$ acceleration $[14,22]$.

For instance, it has been documented that, in a sample of children aged 4 - 5 [14], HR decreased at the start of the sad story, but increased as the story progressed. HR deceleration had begun before the sadness was expressed, leading the researchers to conclude that initial deceleration was indicative of attention to a novel stimulus rather than to a particular mood, whilst subsequent acceleration was indicative of an empathic reaction. It was further found that $\mathrm{HR}$ acceleration during sadness induction was predictive of empathic concern, emotional arousal and prosocial behavior expressed in a different context and time [14]. Interestingly, in studies utilising low intensity sadness contexts, HR deceleration has been associated with consistent prosocial behavior within the immediate context (e.g. proposing to assist the needy person [23], but it failed to predict empathic concern (i.e. sympathy) and prosocial behavior in other situations across time. This might suggest that HR deceleration does not consistently indicate sympathy. Indeed, it should be noted that HR deceleration was not treated as an index of emotional reaction, but solely as an index of other-oriented focus of attention [5].

It seems, then, that HR deceleration during exposure to low intensity sadness may be a reasonable [13], but not an invariant [24] indicator of attention to the affective state of another. It may also be indicative of sympathy, deriving from predominantly cognitive (i.e. apprehension of another's affective state) rather than affective dimensions of empathy, while HR acceleration is a marker of vicariously aroused affect.

However, HR acceleration seems to co-vary with the intensity and type of the stimulus, individual characteristics $[9,25,26]$ and perhaps with the age of the respondents. Several investigators have even gone further to suggest that children's empathy related physiological responding is mediated by factors such as parental expressiveness [13, 27]. HR acceleration should therefore be taken as an imperfect marker of vicariously aroused affect, and caution should be exercised in the interpretation of the results.

For conclusions to be drawn with greater confidence with respect to the effect of stimulus intensity on HR responses, a study manipulating the intensity of the stimulus would give much clearer conclusions rather than various studies that used either high moderate or low stimulus intensity, but, at the same time differed in other aspects which may potentially have had an impact on the differing responses. 
Despite its significant findings, the present investigation should be seen in the context of certain limitations. The capacity for vicarious responsiveness was assessed on a single occasion, using a preselected emotional stimulus, and it remains to be seen whether these results can be replicated across a number of situations and with the use of alternative sets of emotionally evocative stimuli and in different cultural groups.

Further the measure of facial expressions of emotional experience that was used has documented validity. Nonetheless, to an extent it is potentially compromised by a degree of subjectivity inherent in the scoring procedures. For instance the relatively modest emotional intensity that can ethically be induced in an experimental setting may limit the specificity and discriminability of the elicited facial expression [28]. It has been argued that in intensities that are not high the observer might experience an emotion but produce only subvisible changes in the face, rather than full facial prototypes of primary emotions [29].

The methodological limitations of the facial measure used in the current study might be overcome with the use of more refined methodologies, such as the direct recording of facial electromyographic (EMG) activity in facial muscles. With the use of such methodology, Dimberg and colleagues [30] revealed that exposure to emotional facial expressions results in spontaneous EMG reactions in emotion relevant facial muscles. An even further advancement to the field could be made by a newly developed computer-based automatic facial expression analyser [31]. This methodology is in its initial stages of development but, if it provides substantial evidence of validity and reliability, it will circumvent the limitations inherent in the subjective coding and be able to detect even subtle changes of the facial expression.

Such refined methodologies could cast some light to a particular finding of the present investigation. In the episode depicting happiness HR responses were higher relative to baseline not only in the cases when a congruent facial response was recorded. HR responses were higher relative to baseline also in the trials in which no congruent facial response was recorded. Although other explanations are possible (i.e. masking of facial expression of emotion, subtle change in facial expression) it cannot be ruled out that the increase in HR especially in the particular episode might be, at least partly, indicative of increased interest rather than of vicarious affective response.

In conclusion, present findings demonstrate that HR can be used as a consistent marker of vicariously aroused affect in children, at least in the examined age range, in response to others' fear, anger, sadness and the positive emotion of happiness, when these emotional stimuli are of at least moderate intensity.

These findings have substantial implications for studying the typical and atypical development of empathy. For instance, studies of the developmental origins of psychopathy could be enhanced by looking into the psychophysiological characteristics of the child who fails to develop typical empathic responses. Furthermore, these findings have substantial theoretical implications for the empathy literature. If HR increase is interpreted as indicative of vicariously aroused affect [6] present data provide suggestive evidence that, until the age of 10 at least, the affective dimension of empathy predominates over the cognitive when the intensity of stimulus is at least moderate.

\section{ACKNOWLEDGEMENTS}

The authors gratefully acknowledge Dr Marc Obonsawin for his guidance through the physiological assessments, $\mathrm{Dr}$ Bill Cheyne for his statistical advice, all participating children and their parents without whom this study would have been impossible. This research was funded by a University of Strathclyde Research Grant.

\section{REFERENCES}

[1] Cacioppo JT, Sandman CA. Physiological differentiation of sensory and cognitive tasks as a function of warning processing demands and reported unpleasantness. Biol Psychol 1978; 6: 181-92.

[2] Lazarus RS. A cognitively oriented psychologist looks at biofeedback. Am Psychol 1974; 30: 553-61.

[3] Vaughn B, Sroufe LA. The temporal relationship between infant heart rate acceleration and crying in aversive situation. Child Dev 1979; 50: 319-30.

[4] Kagan J. Heart rate and heart rate variability as signs of a temperamental dimensions in infants. In Izard CE, Ed. Measuring Emotions in Infants and Children. Cambridge, Cambridge University Press 1982; 38-66.

[5] Eisenberg N, Fabes RA, Schaller M, Carlo G, Miller PA. The relations of parental characteristics and practices to children's vicarious emotional responding. Child Dev 1991; 62: 1393-408.

[6] Hoffman ML. The measurement of empathy. In. Izard CE, Ed. Measuring emotions in infants and children. Cambridge, Cambridge University Press 1982; 279-96.

[7] Gaertner SL, Dovidio JF. The subtlety of white racism, arousal and helping behaviour. J Pers Soc Psychol 1977; 35: 691-707.

[8] Calkins SD, Dedmon SE. Physiological and behavioural regulation in two year-old children with aggressive/destructive behaviour problems. J Abnorm Child Psychol 2000; 28 (2); 103-18.

[9] Calkins SD, Graziano PA, Keane SP. Cardiac vagal regulation differentiates among children at risk for behavior problems. Biol Psychol 2007; 74(2): 144-53.

[10] Eisenberg N, Fabes RA, Bustamante D, Mathy RM, Miller P A, Lindholm E. Differentiation of vicariously induced reactions in children. Dev Psychol 1988; 24 (2): 237-47.

[11] Eisenberg N, Schaller M, Fabes RA, et al. The differentiation of personal distress and sympathy in children and adults. Dev Psychol 1988; 24: 766-75.

[12] Etzel E, Johnsen J, Dickerson D. Tranel R Adolphs. Cardiovascular and respiratory responses during musical mood induction. Int $\mathrm{J}$ Psychophysiol 2006; 61(1): 57-69.

[13] Liew J, Eisenberg N, Losoya SH, Fabes RA, Guthrie IK, Murphy BC. Children's physiological indices of empathy and their socioemotional adjustment: Does caregivers' expressivity matters. J Fam Psychol 2003; 17 (4): 584-97.

[14] Zahn-Waxler C, Cole PM, Welsh JD, Fox NA. Psychophysiological correlates of empathy and prosocial behaviors in preschool children with problem behaviors. Dev Psychopathol 1995; 7: 2748.

[15] Schwartz GE. Psychophysiological patterning and emotion revisited: a systems perspective. In Izard CE, Ed. Measuring emotions in infants and children. Cambridge: Cambridge University Press 1986; 67-93.

[16] Hauser RM, Featherman DL. The process of stratification. New York: Academic Press 1977.

[17] Carlton TV. Who do you think you are [film]. London, Health \& Social Issues 1982.

[18] Ekman P, Friesen WV. Unmasking the Face. Englewood Clifts NJ: Prentice-Hall 1975.

[19] Izard C, Dougherty L, Hembree E. A system for identifying affect expression by holistic judgment (AFFEX). Newark: University of Delaware. International Resources Center 1983.

[20] Yarczower M, Daruns L. Social inhibition of spontaneous facial expressions in children. J Pers Soc Psychol 1982; 43: 831-7. 
[21] Eisenberg N, McCreath H, Ahn R. Vicarious emotional responsiveness and prosocial behaviour. Their interrelations in young children. Pers Soc Psychol Bull 1988; 14(2): 298-311.

[22] Sánchez-Navarro JP, Martínez-Selva JM, Román F. Uncovering the relationship between defence and orienting in emotion: cardiac reactivity to unpleasant pictures. Int J Psychophysiol 2006; 61(1): 34-46.

[23] Eisenberg N, Fabes RA, Miller PA, Shell R, Shea R, May-Plumlee T. Preschoolers vicarious emotional responding and their situational and dispositional prosocial behaviour. Merrill Palmer Q 1990; 36: 507-29.

[24] Cacioppo JT, Tassinary LG. Inferring psychological significance from physiological signals. Am Psychol 1990; 45: 16-28.

[25] Buck R. The communication of emotion. New York: Guilford Press 1984.

[26] Popma A, Jansen LM, Vermeiren R, et al. Hypothalamus pituitary adrenal axis and autonomic activity during stress in delinquent male adolescents and controls. Psychoneuroendocrinology 2006; 31(8): 948-57.
Gilissen R, Koolstra CM, van Ijzendoorn MH, BakermansKranenburg MJ, van der Veer R. Physiological reactions of preschoolers to fear-inducing film clips: effects of temperamental fearfulness and quality of the parent-child relationship. Dev Psychobiol 2007; 49(2): 187-95.

[28] Fabes RA, Eisenberg N, Miller PA. Maternal correlates of children's vicarious emotional responsiveness. Dev Psychol 1990; 26: 639-48.

[29] Levenson RW. Emotion and the autonomic nervous system: a prospectus for research on autonomic specificity. In Wagner HL, Ed. Social psychophysiology and Emotion: Theory and clinical application (pp.19-42) John Wiley and Sons: New York 1988.

[30] Dimberg U, Thunberg M, Elmehed K. Unconscious facial reactions to emotional facial expressions. Psychol Sci 2000; 11: 86-9.

[31] Pantic M, Rothkrantz LJM. Automatic analysis of facial expressions: the state of the art. Anal Mach Intell 2000; 22: 1424-2445.

(C) Anastassiou-Hadjicharalambous and Warden; Licensee Bentham Open.

This is an open access article licensed under the terms of the Creative Commons Attribution Non-Commercial License (http: //creativecommons.org/licenses/ by-nc/3.0/) which permits unrestricted, non-commercial use, distribution and reproduction in any medium, provided the work is properly cited. 